Europhysics Letters

PREPRINT

\title{
On the formation/dissolution of equilibrium droplets
}

\author{
M. BISKUP ${ }^{1}$, L. CHAYES ${ }^{1}$ and R. KoteckÝ ${ }^{2}$ \\ 1 Department of Mathematics, UCLA, Los Angeles, California 90095-1555, USA \\ 2 Center for Theoretical Study, Charles University, Jilská 1, 11000 Prague, Czech Republic \\ PACS. 05.70.Fh - Phase transitions: general studies. \\ PACS. 64.60.Cn - Order-disorder transformations, statistical mechanics of model systems. \\ PACS. $75.10 . \mathrm{Hk}-$ Classical spin models.
}

\begin{abstract}
We consider liquid-vapor systems in finite volume $V \subset \mathbb{R}^{d}$ at parameter values corresponding to phase coexistence and study droplet formation due to a fixed excess $\delta N$ of particles above the ambient gas density. We identify a dimensionless parameter $\Delta \sim(\delta N)^{(d+1) / d} / V$ and a universal value $\Delta_{\mathrm{c}}=$ $\Delta_{\mathrm{c}}(d)$, and show that a droplet of the dense phase occurs whenever $\Delta>\Delta_{\mathrm{c}}$, while, for $\Delta<\Delta_{\mathrm{c}}$, the excess is entirely absorbed into the gaseous background. When the droplet first forms, it comprises a non-trivial, universal fraction of excess particles. Similar reasoning applies to generic two-phase systems at phase coexistence including solid/gas — where the "droplet" is crystalline-and polymorphic systems. A sketch of a rigorous proof for the 2D Ising lattice gas is presented; generalizations are discussed heuristically.
\end{abstract}

Introduction. - The thermodynamics of droplets in systems with phase coexistence has been well understood since the pioneering works [1] 4 . Recently, justifications of the classic results based on the first principles of statistical mechanics have been attempted — in both two [5-9] and higher [10 13] dimensions - and various thermodynamical predictions concerning macroscopic shapes have been rigorously established. However, the formation and dissolution of equilibrium droplets is among the less well-studied areas in statistical mechanics. Indeed, most of the aforementioned analysis has focused on the situation implicitly assumed in the classical derivations; namely, that the scale of the droplet is comparable with the scale of the system. As is known [7, 8, 14 [16], this will not be the case when the parameter values are such that a droplet first forms. In this Letter, we underscore the region of the system parameters that is critical for the formation/dissolution of droplets. In particular, we isolate the mechanism by which the low-density phase copes with an excess of particles and pinpoint the critical amount of extra particles needed to cause a droplet to appear. Surprisingly, at the point of droplet formation, only a certain fraction of the excess goes into the droplet; the rest is absorbed by the bulk. Moreover, apart from a natural rescaling to dimensionless parameters, all of the above can be described in terms of universal quantities independent of the system particulars and the temperature.

In the last few years, there has been some interest in questions related to droplet formation and dissolution with purported applicability in diverse areas such as nuclear fragmentation [17-19] and the stability of adatom islands on crystal surfaces [15, 20]. Another issue, which is of practical significance in statistical mechanics, concerns the detection of first-order phase transitions by the study of small systems with fixed order parameter (magnetization) or fixed energy. Under these conditions, nonconvexities appear in the finite-volume thermodynamic functions (which, of course, must vanish in

(C) EDP Sciences 
the thermodynamic limit), see [21-24]. Naturally, this suggests the formation of a droplet in a system with coexisting phases. Several studies have directly addressed the issues surrounding the appearance of droplets with intriguing reports on finite-size characteristics [15, 20, 21, 25, 26]. We believe that the results of this Letter may shed some light in these situations.

Droplets in systems at phase coexistence. - We will start with some general considerations which bolster the claims of the first paragraph and, at the end of this Letter, describe the principal steps of a rigorous proof for the 2D Ising lattice gas. Although the natural setting for these problems is the canonical distribution in finite volume, intuition is often better developed in the context of finite subsystems using the language of the grandcanonical ensemble. Here the occurrence of droplets may be regarded as a problem in large deviation theory. This perspective will guide our heuristic analysis as it did in the proof for the 2D Ising system.

Consider a generic liquid-vapor system. (In this Letter, we adopt, for concreteness, the language of the liquid-vapor transition. However, all considerations apply equally well to the formation/dissolution of an equlibrium crystal against a liquid or gaseous background.) First, suppose that the system is in the gaseous phase. According to a fluctuation-dissipation analysis, the local fluctuations for subsystems of volume $V$ are then of the order $\sqrt{\varkappa V}$, where, modulo constants, $\varkappa$ is the isothermal compressibility. More precisely, the probability of observing a particle excess $\delta N$ is given by

$$
\exp \left\{-\frac{(\delta N)^{2}}{2 \varkappa V}\right\}
$$

Now, when $\delta N=\mathcal{O}(\sqrt{\varkappa V})$, the above is just the leading-order asymptotic of a full-fledged Gaussian (central limit) distribution, which comes equipped with power-law corrections, etc. Moreover-in the single phase regime - the above leading order remains valid even for $(\delta N)^{2} \gg \varkappa V$, provided that $|\delta N| \ll \rho_{\mathrm{G}} V$, where $\rho_{\mathrm{G}}$ is the gas density.

In the two-phase regime, small excesses can be again absorbed into background fluctuations but, in addition, a second mechanism exists through which the system can handle an excess of particles; namely, the formation of liquid-phase droplets. The minimal cost of a droplet of volume $\delta V$ goes as

$$
\exp \left\{-\tau_{\mathrm{W}}(\delta V)^{\frac{d-1}{d}}\right\}
$$

where $\tau_{\mathrm{W}}$ denotes the (surface) free energy of an ideal-shape droplet of unit volume. For an isotropic system, $\tau_{\mathrm{W}}=\tau S_{d}$, where $\tau$ is the surface tension and $S_{d}=2 \pi^{d / 2} / \Gamma\left(\frac{d}{2}\right)$ is the surface area of the unit sphere in $\mathbb{R}^{d}$. In general, $\tau_{\mathrm{W}}$ is obtained by minimizing the Wulff functional [ $[3]$. As noted already in [1] 3 ], by isoperimetric inequalities, scenarios involving multiple macroscopic droplets are far less likely.

Now the number of excess particles $\delta N$ in a droplet of volume $\delta V$ is just $\delta N=\left(\rho_{\mathrm{L}}-\rho_{\mathrm{G}}\right) \delta V$, where $\rho_{\mathrm{G}}$ and $\rho_{\mathrm{L}}$ are the ambient gas and liquid densities, respectively. Thus, comparing Eqs. (1) and (2), the droplet mechanism dominates when $\delta N \gg \Theta V^{d /(d+1)}$, where $\Theta^{d+1}=\left(\varkappa \tau_{\mathrm{W}}\right)^{d}\left(\rho_{\mathrm{L}}-\right.$ $\left.\rho_{\mathrm{G}}\right)^{1-d}$, while the fluctuation mechanism dominates when $\delta N \ll \Theta V^{d /(d+1)}$. We note that, from the perspective of rigorous analysis, significant progress has been made in the single-phase regime and in the above-mentioned extreme cases of the two-phase regime. For the Ising model, this was done for low temperatures in the exhaustive paper [7], while [8] extended this result throughout the coexistence region in the case $d=2$.

Previously, the crossover region $\delta N \approx \Theta V^{d /(d+1)}$ has not received adequate attention. To study this region, we introduce the appropriate dimensionless parameter

$$
\Delta=\frac{\left(\rho_{\mathrm{L}}-\rho_{\mathrm{G}}\right)^{\frac{d-1}{d}}}{2 \varkappa \tau_{\mathrm{W}}} \frac{(\delta N)^{\frac{d+1}{d}}}{V}
$$


and investigate finite (but large) size systems as $\Delta$ varies. The key to the whole picture is that, for the entire range of $\Delta$, there is a forbidden interval of droplet sizes. To be precise, let us categorize droplets according to their surface area: We will say a droplet is of intermediate size if its surface area is large compared with $\log V$ but small compared with $V^{(d-1) /(d+1)}$. Droplets with surface areas outside this range will be called large and small as appropriate. We will show that, with overwhelming probability, there are no intermediate droplets.

We begin with some observations: Suppose we specify the amount of excess which goes into intermediate and large scale droplets and fix the location of these droplets. Then, throughout the rest of the system, the fluctuations-dissipation result in Eq. (11) is valid, at least to leading order. Indeed, the only obstructions to Gaussian-type bulk fluctuations are: (1) The appearance of droplets beyond the logarithmic scale, which we have already separated for a special treatment, and (2) An exorbitant surface to volume ratio. Since $\delta N \approx \Theta V^{d /(d+1)}$ (i.e., $\left.\Delta<\infty\right)$, the second possibility does not occur. Let

$$
\delta N=\delta N_{\mathrm{L}}+\delta N_{\mathrm{I}}+\delta N_{\mathrm{S}}
$$

where $\delta N_{\mathrm{L}}$ is the amount of excess particles in large droplets, and similarly for $\delta N_{\mathrm{I}}$ and $\delta N_{\mathrm{S}}$. By Eq. (1), the distribution of the excess given in Eq. (4) has a cost $\exp \left\{-\left(\delta N_{\mathrm{S}}\right)^{2} /(2 \varkappa V)\right\}$ for the fluctuation part. The cost of intermediate droplets will be of the order of their combined surface. If there are $n$ such droplets, then isoperimetric reasoning forces us to pay at least $\exp \left\{-\tau_{\mathrm{W}} C\left(\delta N_{\mathrm{I}}\right)^{(d-1) / d} n^{1 / d}\right\}$, where $C$ is a constant of order unity. On the other hand, if all of $\delta N_{\mathrm{I}}$ were to go into small scale fluctuations, we would simply have to pay $\exp \left\{-\left(\delta N_{\mathrm{S}}+\delta N_{\mathrm{I}}\right)^{2} /(2 \varkappa V)\right\}$. Comparing the two mechanisms we find, using $\delta N_{\mathrm{S}} \lesssim \Theta V^{d /(d+1)}$ and $\delta N_{\mathrm{I}} \ll n \Theta V^{d /(d+1)}$, that

$$
\frac{\left(\delta N_{\mathrm{S}}\right)^{2}}{2 \varkappa V}+\tau_{\mathrm{W}} C\left(\delta N_{\mathrm{I}}\right)^{\frac{d-1}{d}} n^{1 / d} \gg \frac{\left(\delta N_{\mathrm{S}}+\delta N_{\mathrm{I}}\right)^{2}}{2 \varkappa V},
$$

whenever $n \geq 1$. Hence, the probability of even a single droplet of the intermediate scale is utterly negligible.

Having established the absence of intermediate-scale droplets, isoperimetric inequalities rule out the possibility of more than one large droplet. Thus, we are down to the simplest possible scenario: There is (at most) a single large droplet in the system - the cost of which is governed by Eq. (2) absorbing some of the excess, while the rest goes into background fluctuations-which are described by Eq. (11). Thus, the probability that the droplet contains the fraction $\lambda$ of the excess particles is, in the leading order, given by

$$
\exp \left\{-\tau_{\mathrm{W}}\left(\frac{\delta N}{\rho_{\mathrm{L}}-\rho_{\mathrm{G}}}\right)^{\frac{d-1}{d}} \Phi_{\Delta}(\lambda)\right\}
$$

where $\Phi_{\Delta}(\lambda)$ is defined by

$$
\Phi_{\Delta}(\lambda)=\lambda^{\frac{d-1}{d}}+\Delta(1-\lambda)^{2} .
$$

In particular, with overwhelming probability, the fraction of excess particles taken by the droplet corresponds to a value of $\lambda$ that minimizes $\Phi_{\Delta}(\lambda)$.

The result of a straightforward computation is that there is a constant $\Delta_{c}$, given by the expression

$$
\Delta_{\mathrm{c}}=\frac{1}{d}\left(\frac{d+1}{2}\right)^{\frac{d+1}{d}},
$$

which separates two types of behavior: For $\Delta<\Delta_{c}$, the unique global minimizer of $\Phi_{\Delta}(\lambda)$ is $\lambda=0$, while for $\Delta>\Delta_{\mathrm{c}}$, the unique global minimum of $\Phi_{\Delta}$ occurs at a non-trivial value $\lambda_{\Delta}>0$, see Fig. 11. 

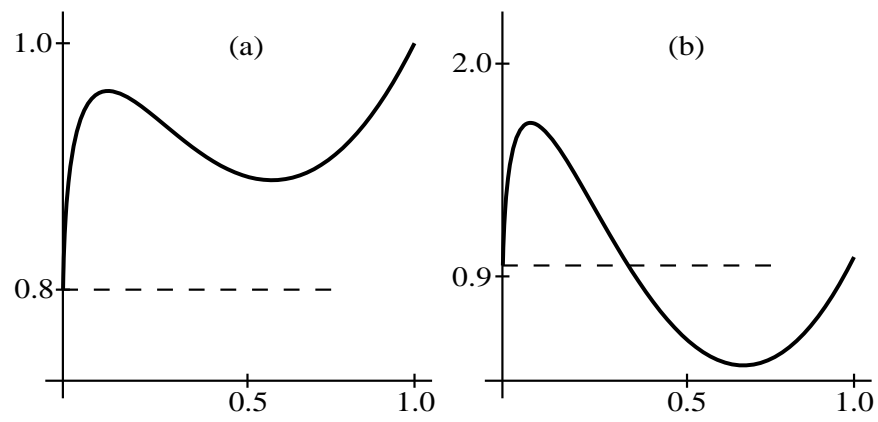

Fig. 1 - The graph of the universal function $\Phi_{\Delta}$ in $d=2$. Here the parameter $\lambda$ represents the trial fraction of the excess that goes into the droplet; $\Phi_{\Delta}$ has the interpretation of a free energy function. In (a), $\Delta=0.8<\Delta_{\mathrm{c}}$, and the function is minimized by $\lambda=0$. In (b), $\Delta=0.96>\Delta_{\text {c }}$ and the function is minimized by a $\lambda=\lambda_{\Delta}>2 / 3$. The maximum that interdicts between $\lambda=0$ and $\lambda=\lambda_{\Delta}$ presumably plays the role of a free energy barrier for the formation of the droplet.

Moreover, the quantity $\lambda_{\Delta}$ increases monotonically with $\Delta$ and the value of $\lambda_{\Delta}$ at $\Delta=\Delta_{c}$, denoted by $\lambda_{\mathrm{c}}$, can be computed exactly;

$$
\lambda_{\mathrm{c}}=\frac{2}{d+1} .
$$

In particular, we have $\lambda_{\Delta} \geq \lambda_{\mathrm{c}}$ for all $\Delta \geq \Delta_{\mathrm{c}}$. See Fig. 目.

Let us interpret the results in the context of the canonical distribution: The region $\Delta<\Delta_{\mathrm{c}} \min -$ imized by $\lambda \equiv 0$ is the remnant of the "phase" $\delta N \ll \Theta V^{d /(d+1)}$; the entire excess is taken up by background fluctuations. For $\Delta>\Delta_{c}$, a large droplet occurs which absorbs the fraction $\lambda_{\Delta}$ of the particle excess. Although this is obviously a precursor to the droplet-dominated "phase" (where $\left.\delta N \gg \Theta V^{d /(d+1)}\right)$, the physics is somewhat different since a finite fraction of the excess-namely $\left(1-\lambda_{\Delta}\right) \delta N$ particles-is still handled by the background. We emphasize that $\lambda_{\Delta}$ and $\Delta$ are related via a simple algebraic equation. The system-specific details and dependence on external parameters are encoded into the factor $\left(\rho_{\mathrm{L}}-\rho_{\mathrm{G}}\right)^{(1-d) / d} \varkappa \tau_{\mathrm{W}}$ from Eq. (3); the dimensionless parameter $\lambda_{\Delta}$ is a universal function of $\Delta$.

We remark that in [14 16], similar conclusions had been reached by various circuitous routes under the mantel of specialized assumptions or approximations. In this note, the exact formula has been derived on the basis of simple-minded droplet/fluctuation-dissipation arguments, all of which can be rigorously proved in at least one case.

Mathematical results for 2D Ising model. - In the context of the two dimensional Ising lattice gas, the above reasoning has been elevated to the status of a mathematical theorem, which for convenience we state in the language of the equivalent spin system. Consider the square-lattice Ising model with the (formal) Hamiltonian

$$
\mathcal{H}=-\sum_{\langle x, y\rangle} \sigma_{x} \sigma_{y}
$$

where $\sigma_{x}= \pm 1$ and $\langle x, y\rangle$ denotes a nearest-neighbor pair. For each inverse temperature $\beta$, let $m^{\star}=$ $m^{\star}(\beta)$ be the spontaneous magnetization, $\chi=\chi(\beta)$ the magnetic susceptibility, and $\tau_{\mathrm{W}}=\tau_{\mathrm{W}}(\beta)$ be the minimal value of the Wulff functional for droplets of unit volume. As is well known, $m^{\star}(\beta)>0$, $0<\chi(\beta)<\infty$ and $\tau_{\mathrm{W}}(\beta)>0$ once $\beta>\beta_{\mathrm{c}}=\frac{1}{2} \log (1+\sqrt{2})$. 


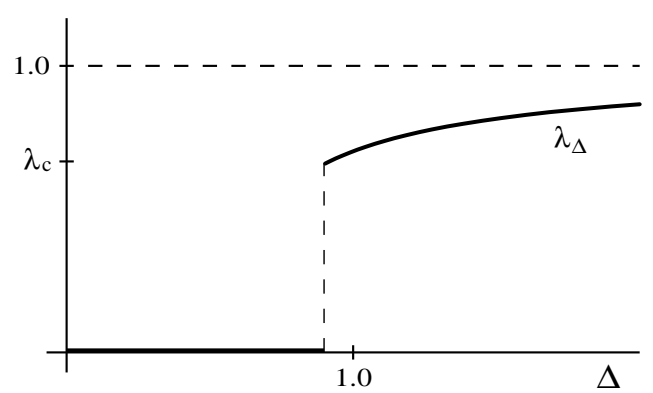

Fig. 2 - The graph of $\lambda_{\Delta}$, the fraction of the excess that goes into the droplet, as a function of $\Delta$, the dimensionless rescaled parameter that measures the total excess. Here $d=2$. Notice that $\lambda_{\Delta}=0$ for $\Delta<\Delta_{\mathrm{c}} \approx 0.918$, but as $\Delta \downarrow \Delta_{\mathrm{c}}, \lambda_{\Delta}$ tends to $\lambda_{\mathrm{c}}=2 / 3$. The behavior of the system at $\Delta=\Delta_{\mathrm{c}}$ has not been fully elucidated.

Consider now an $L \times L$ square in $\mathbb{Z}^{2}$ denoted by $\Lambda_{L}$ and let $M_{L}=\sum_{x \in \Lambda_{L}} \sigma_{x}$ be the overall magnetization in $\Lambda_{L}$. Let $v_{L} \geq 0$ be such that $m^{\star}\left|\Lambda_{L}\right|-2 m^{\star} v_{L}$ is an allowed value of $M_{L}$ for all $L$. Let $P_{L, v_{L}}^{+, \beta}$ be the canonical distribution on $\Lambda_{L}$ with plus boundary conditions, inverse temperature $\beta$, and $M_{L}$ fixed to the value $m^{\star}\left|\Lambda_{L}\right|-2 m^{\star} v_{L}$. In the present setting, the parameter $\Delta$ in Eq. (3) becomes

$$
\Delta=2 \frac{\left(m^{\star}\right)^{2}}{\chi \tau_{\mathrm{W}}} \lim _{L \rightarrow \infty} \frac{v_{L}^{3 / 2}}{\left|\Lambda_{L}\right|}
$$

where we presume that the limit exists.

In Ising systems, a convenient description for the spin configurations is in terms of their Peierls' contours, i.e., the lines separating spins of opposite type; see Fig. 3. Moreover, in the present context, the boundaries of droplets are exactly these contour lines. Our first claim concerns the absence of contours of intermediate size, regardless of the value of $\Delta$.

Theorem I. Let $\beta>\beta_{\mathrm{c}}$ and suppose that the limit in Eq. (11) exists with $\Delta \in(0, \infty)$. Let $\mathcal{A}_{L}$ be the event that there is no contour $\Gamma$ in $\Lambda_{L}$ with

$$
K \log L \leq \operatorname{diam} \Gamma \leq \frac{1}{K} L^{2 / 3} .
$$

If $K=K(\beta)$ is sufficiently large, then

$$
\lim _{L \rightarrow \infty} P_{L, v_{L}}^{+, \beta}\left(\mathcal{A}_{L}\right)=1
$$

We remark that this is the rigorous (albeit 2D-Ising specific) analogue of our general argument in Eq. (3)-Eq. (5). The above theorem is far and away the most difficult part of the mathematical analysis. Notwithstanding, the flow of the proof parallels closely the derivation that was given here. The reader may have noticed that, in the present derivation, we have not used in any obvious way the lower bound defining the scale of intermediate droplets. The issue is somewhat delicate since some log-scale droplets will naturally emerge from the background fluctuations. The constant $K$ must be chosen large in order to enforce the distinction between "natural" and "unnatural" as well as to control the translation entropy of the purported intermediate droplets.

Our next goal is to specify the typical configurations in measure $P_{L, v_{L}}^{+, \beta}$ depending on the value $\Delta$ as compared with $\Delta_{\mathrm{c}}$. Let $\mathcal{B}_{L, K}$ be the event that there is no contour $\Gamma$ in $\Lambda_{L}$ with diam $\Gamma \geq K \log L$. Furthermore, let $\mathcal{C}_{L, K}$ be the event that there is one contour $\Gamma_{0}$ with diam $\Gamma_{0} \geq \frac{1}{K} L^{2 / 3}$ while all other contours $\Gamma$ in $\Lambda_{L}$ satisfy diam $\Gamma \leq K \log L$. 


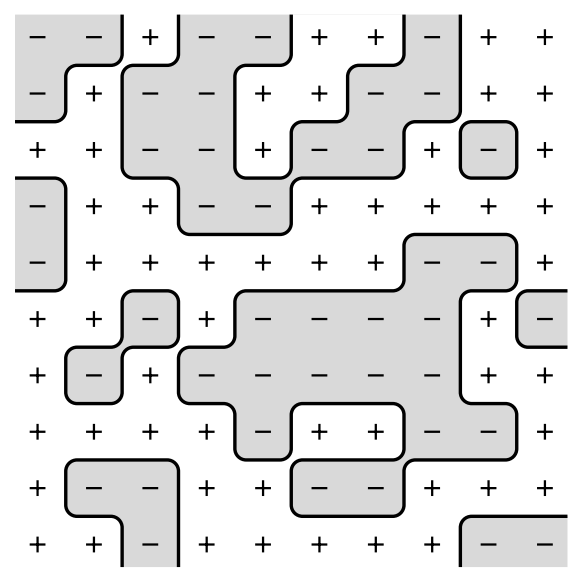

Fig. 3 - Peierls' contours in a 2D-Ising spin configuration. In the lattice-gas language, we regard minus spins as particles and plus spins as vacancies. Peierls' contours are then the interfaces separating high and low density regions at the microscopic scale.

Theorem II. Let $\beta>\beta_{\mathrm{c}}$ and suppose that the limit in Eq. (11) exists with $\Delta \in(0, \infty)$. Let $\Delta_{\mathrm{c}}$ be as in Eq. (8) and, for $\Delta>\Delta_{c}$, let $\lambda_{\Delta}$ be the unique minimizer of $\Phi_{\Delta}(\lambda)$.

(1) If $\Delta<\Delta_{\mathrm{c}}$ and $K=K(\beta)$ is sufficiently large, then

$$
\lim _{L \rightarrow \infty} P_{L, v_{L}}^{+, \beta}\left(\mathcal{B}_{L, K}\right)=1
$$

(2) If $\Delta>\Delta_{\mathrm{c}}$ and $K=K(\beta)$ is sufficiently large, then

$$
\lim _{L \rightarrow \infty} P_{L, v_{L}}^{+, \beta}\left(\mathcal{C}_{L, K}\right)=1 .
$$

Moreover, with probability approaching one, the unique "large" contour $\Gamma_{0}$ has volume $\left(\lambda_{\Delta}+o(1)\right) v_{L}$ and its shape asymptotically optimizes the surface-energy (Wulff) functional for the given volume.

Theorems I and II completely classify the behavior of the Ising system for all $\Delta \neq \Delta_{\mathrm{c}}$. We emphasize that the situation at $\Delta=\Delta_{\mathrm{c}}$ has not been fully clarified. What can be ruled out, according to Theorem I, is the possibility of a complicated scenario involving intermediate size droplets on a multitude of scales. Indeed, when $\Delta=\Delta_{\mathrm{c}}$, in (almost) every configuration we must have either a single large droplet or no droplet at all; i.e., the outcome must mimic the case $\Delta>\Delta_{\mathrm{c}}$ or $\Delta<\Delta_{\mathrm{c}}$. It is conceivable that one outcome dominates all configurations or that both outcomes are possible depending on auxiliary conditions.

Our last statement concerns the decay of the probability (in the grandcanonical distribution) that the overall magnetization takes value $m^{\star}\left|\Lambda_{L}\right|-2 m^{\star} v_{L}$. Let $\mathcal{P}_{L}^{+, \beta}$ be the (Gibbs) probability distribution on spins in $\Lambda_{L}$ with plus boundary condition and inverse temperature $\beta$.

Theorem III. Let $\beta>\beta_{\mathrm{c}}$ and suppose that the limit in $E q$. (11) exists with $\Delta \in(0, \infty)$. Introduce the shorthand $p_{L}=\mathcal{P}_{L}^{+, \beta}\left(M_{L}=m^{\star}\left|\Lambda_{L}\right|-2 m^{\star} v_{L}\right)$. Then

$$
\lim _{L \rightarrow \infty} v_{L}^{-1 / 2} \log p_{L}=-\tau_{\mathrm{W}} \inf _{0 \leq \lambda \leq 1} \Phi_{\Delta}(\lambda) .
$$


Sketch of the proofs. - Here we outline the steps necessary to prove the above theorems. As already noted, first we reduce the problem to the study of large-deviation properties of the "grandcanonical" distribution $\mathcal{P}_{L}^{+, \beta}$ using the relation

$$
P_{L, v_{L}}^{+, \beta}(\mathcal{A})=\mathcal{P}_{L}^{+, \beta}\left(\mathcal{A}\left|M_{L}=m^{\star}\right| \Lambda_{L} \mid-2 m^{\star} v_{L}\right),
$$

valid for all events $\mathcal{A}$. The next technical step is then a proof of the large-deviation lower bound

$$
p_{L} \geq \exp \left\{-\tau_{\mathrm{W}} \sqrt{v_{L}}\left(\Phi_{\Delta}(\lambda)+\epsilon_{L}\right)\right\},
$$

which is produced by forcing in a contour of the appropriate size and evaluating the contributions from surface tension and bulk fluctuations. Here $\epsilon_{L} \rightarrow 0$ as $L \rightarrow \infty$, uniformly in $\lambda$. A comparison with this lower bound then shows that, with overwhelming probability in $P_{L, v_{L}}^{+, \beta}$, the total surface area of contours $\Gamma$ with diam $\Gamma \geq K \log L$ is at most of order $\sqrt{v_{L}}$ while their combined volume is at most of order $v_{L}$. This puts us in a position to carry out the argument in Eq. (5), which ultimately leads to the proof of Theorem I. Having eliminated the intermediate contours, we are down to the scenario with at most one large contour. Optimizing over the contour volume/shape proves Theorem II and also produces a large-deviation upper bound, which completes the proof of Theorem III.

Theorems I-III pretty much tell the story for this particular case. Complete proofs and additional details will appear elsewhere [27].

The research of R.K. was partly supported by the grants GAČR 201/00/1149 and MSM 110000001. The research of L.C. was supported by the NSF under the grant DMS-9971016 and by the NSA under the grant NSA-MDA 904-00-1-0050.

\section{REFERENCES}

[1] GibBs J.W., Gibbs Collected Works (Yale Univ. Press, New Haven, Conn.) 1928

[2] Curie P., Bull. Soc. Fr. Mineral., 8 (1885) 145

[3] WulfF G., Z. Krystallog. Mineral., 34 (1901) 449

[4] LAngmuir I., The collected works of Irving Langmuir (Surface phenomena), Vol. 9 (Pergamon Press, New York) 1960

[5] Alexander K., Chayes J. T. and Chayes L., Commun. Math. Phys., 131 (1990) 1

[6] Dobrushin R. L., KoteckÝ R. and Shlosman S. B., Wulff construction. A global shape from local interaction, (Amer. Math. Soc., Providence, RI) 1992

[7] Dobrushin R. L. and Shlosman S. B., Probability contributions to statistical mechanics, pp. 91-219 (Amer. Math. Soc., Providence, RI) 1994

[8] Ioffe D. and Schonmann R. H., Commun. Math. Phys., 199 (1998) 117

[9] Pfister C.-E. and Velenik Y., Commun. Math. Phys., 204 (1999) 269

[10] CERF R., Astérisque, 267 (2000) vi+177

[11] Bodineau T., Commun. Math. Phys., 207 (1999) 197

[12] Cerf R. and Pisztora A., Ann. Probab., 28 (2000) 947

[13] Bodineau T., Ioffe D. and Velenik Y., J. Math. Phys., 41 (2000) 1033

[14] Binder, K. and Kalos, M. H., J. Statist. Phys, 22 (1980) 363

[15] Krishnamachari B., Mclean J., Cooper B. and Sethna J., Phys. Rev. B, 54 (1996) 8899

[16] Neuhaus T. and Hager J. S., cond-mat/0201324.

[17] Carmona J.M., Richert J. and Tarancón A., Nucl. Phys. A, 643 (1998) 115

[18] Pan J., Das Gupta S. and Grant M., Phys. Rev. Lett, 80 (1998) 1182

[19] Gulminelli F. and Chomaz Ph., Phys. Rev. Lett, 82 (1999) 1402 
[20] Müller T. and Selke W., Eur. Phys. J. B, 10 (1999) 549

[21] Pleimling M. and Hüller A., J. Statist. Phys., 104 (2001) 971

[22] Gross D. H. E., Microcanonical Thermodynamics: Phase Transitions in "Small” Systems (Lecture Notes in Physics), Vol. 66 (World Scientific, Singapore) 2001

[23] Machta J., Choi Y.S., Lucke A., Schweizer T. and Chayes L. M., Phys. Rev. E, 54 (1996) 1332

[24] Lee J. and Kosterlitz J. M., Phys. Rev. B, 43 (1990) 3265

[25] Farukawa, H. and Binder, K., Phys. Rev. A, 26 (1982) 556

[26] Pleimling M. and Selke W., J. Phys. A, 33 (2000) L199

[27] BisKuP M., ChAYES L. and KOTECKÝ R., in preparation. 